\title{
A selective screening platform reveals unique global expression patterns of microRNAs in a cohort of human soft-tissue sarcomas
}

\author{
Peter Y Yu ${ }^{1}$, Mumtaz Y Balkhi 2,3,4, Katherine J Ladner ${ }^{2,3}$, Hansjuerg Alder²,3, Lianbo Y Y ${ }^{5}$, Xiaokui Mo ${ }^{5}$, \\ William G Kraybill ${ }^{2,6}$, Denis C Guttridge ${ }^{2,3}$ and $O$ Hans Iwenofu ${ }^{2,7}$
}

Sarcomas are malignant heterogenous tumors of mesenchymal derivation. Emerging data suggest that miRNA might have a causal role in sarcomagenesis. Herein, we used a selective miRNA screening platform to study the comparative global miRNA expression signatures in a cohort of human sarcomas with the caveat that comparisons between tumor and non-tumor cells were performed from the same patients using formalin-fixed paraffin-embedded tissue. Five histologic types were examined that included: myxoid liposarcoma, well-differentiated liposarcoma, dedifferentiated liposarcoma, pleomorphic rhabdomyosarcoma, and synovial sarcoma. In addition, soft-tissue lipomas and normal fat were included as a separate set of controls for the lipogenic tumors. Clustering analysis showed a distinct global difference in expression patterns between the normal and sarcoma tissues. Expression signatures in an unsupervised hierarchical clustering analysis revealed tight clustering in synovial and myxoid liposarcomas, and the least clustering was observed in the pleomorphic rhabdomyosarcoma subtype. MiR-145 showed underexpression in pleomorphic rhabdomyosarcoma, well-differentiated liposarcoma, and synovial sarcoma. Unexpectedly, we found that a set of muscle-specific microRNAs (miRNAs; myomiRs): miR-133, miR-1, and miR-206 was significantly underexpressed in well-differentiated liposarcoma and synovial sarcoma, suggesting that they may function as tumor suppressors as described in muscle-relevant rhabdomyosarcomas. In addition, a tight linear progression of miRNA expression was identified from normal fat to dedifferentiated liposarcoma. These results suggest that miRNA expression profiles could elucidate classes of miRNAs that may elicit tumor-relevant activities in specific sarcoma subtypes.

Laboratory Investigation (2016) 96, 481-491; doi:10.1038/labinvest.2015.168; published online 15 February 2016

Sarcomas are rare malignant heterogeneous tumors of mesenchymal derivation with over 50 histologic types described. ${ }^{1}$ The pathogenesis and biology of many of the different histologic subtypes remain poorly understood. Recent progress in understanding the biology of sarcomas has identified distinct molecular and pathologic entities within these heterogeneous groups of tumors that have paved the way for development of targeted therapies. There are emerging data that seem to suggest that miRNA might be a driver in sarcomagenesis with potential therapeutic implications.
microRNAs (miRNAs) are non-coding strands of RNA of $\sim 22$ nucleotides in length that regulate the expression of genes involved in processes such as development, differentiation, cell proliferation, metabolism, cell death, viral infection, and cancer. ${ }^{2,3}$ They have a broad effect through base pairing with mRNA at their $3^{\prime}$ untranslated region to inhibit translation or to target the mRNA for degradation. Bioinformatics predictions have shown that miRNAs regulate $\sim 30 \%$ of mammalian protein-coding genes. Their role in the molecular biology of cancers is well-established partly

\footnotetext{
Medical Student Research Program, The Ohio State University College of Medicine, The Ohio State University Wexner Medical Center, Columbus, OH, USA; ${ }^{2}$ Arthur G. James Comprehensive Cancer Center, The Ohio State University Wexner Medical Center, Columbus, OH, USA; ${ }^{3}$ Department of Molecular Virology, Immunology and Medical Genetics, The Ohio State University Medical Center, Columbus, OH, USA; ${ }^{\circ}$ Department of Medicine, Tufts Medical Center and Tufts University School of Medicine, Boston, MA, USA; ${ }^{5}$ Center for Biostatistics, Department of Biomedical Informatics, The Ohio State University Wexner Medical Center, Columbus, OH, USA; ${ }^{6}$ Department of Surgical Oncology, The Ohio State University Wexner Medical Center, Columbus, OH, USA and ${ }^{7}$ Department of Pathology and Laboratory Medicine, The Ohio State University Wexner Medical Center, Columbus, OH, USA Correspondence: Dr DC Guttridge, PhD, Department of Molecular Virology, Immunology and Medical Genetics, The Ohio State University Wexner Medical Center, 520 Biomedical Research Tower, 460 West 12th Avenue, Columbus, OH 43210, USA or Dr OH Iwenofu, MD, Department of Pathology and Laboratory Medicine, The Ohio State University Wexner Medical Center, 410 West 10th Avenue, E-418A Doan Hall, Columbus, OH 43210, USA.
}

E-mail: denis.guttridge@osumc.edu or hans.iwenofu@osumc.edu

Received 1 December 2015; accepted 18 December 2015 
because $>50 \%$ of miRNA genes are located at chromosomal fragile sites, common break point sites, or regions of deletion/amplification that are often altered in human malignancies. ${ }^{4}$ Thus, many miRNAs have been identified to function as either tumor suppressors or oncogenes. ${ }^{5}$ Indeed, recent studies on miRNA expression signatures from hematological and solid tumors appear to discriminate different types of human cancers with high accuracy. ${ }^{6}$ Due to these functions, miRNAs have potential for the diagnosis, prognosis, and therapy of cancer.

Unique miRNA expression signatures have been reported for many of the different classes of sarcomas. ${ }^{7,8}$ These distinct patterns could potentially be used to identify the histologic type of sarcoma. Identification of specific miRNA expression has already begun to elucidate the pathophysiology behind some sarcomas. For example, we and others found that the tumor suppressor miR-29 was found to be downregulated in rhabdomyosarcoma. ${ }^{9,10}$ Results identified a regulatory circuit involving miR-29, NF- $\mathrm{B}$, and YY1 that appears to be dysregulated in rhabdomyosarcoma and might contribute to the dedifferentiation phenotype of these tumors. ${ }^{9}$ Re-expression of miR-29 in xenograft tumors and rhabdomyosarcoma cell lines led to inhibition of tumor proliferation as well as stimulation of myogenic differentiation. Similarly, the muscle-specific miRNA (myomiR), miR-206, is downregulated in rhabdomyosarcoma, ${ }^{11-14}$ and investigators found that re-expression of miR-206 in xenografted tumors stopped proliferation and stimulated myogenic differentiation. ${ }^{11}$ The MET protooncogene has been implicated as a target of miR-206 and was indeed silenced with miR-206 re-expression. ${ }^{11,12}$

Up until recently, most studies on miRNAs in sarcoma have utilized frozen specimens and have used tissue obtained from different patients as normal controls. Given the variability in miRNA expression between different individuals, ${ }^{15-17}$ this methodology may lead to inaccurate results. The relative stability of miRNAs in formalin-fixed paraffin-embedded (FFPE) archival tissue has made them an attractive resource for miRNA profiling studies and indeed has been shown to be comparable to fresh frozen specimens. ${ }^{18}$ Against this background, we had optimized a technique in our recent work using FFPE material where we used the patient's non-tumoral tissue as a means to minimize the variability introduced by using normal tissue controls from different patients. ${ }^{19}$ This was done using a tissue microarrayer to core tumor and non-tumoral areas that had been mapped on hematoxylin and eosin-stained sections so that the tumor samples were matched with paired controls from the same patient. In this current study, we explored miRNA expression in a selected cohort of human soft-tissue sarcomas. Conceptually, we chose to test genetically simple sarcomas (including well-differentiated liposarcoma, myxoid liposarcoma, and synovial sarcoma) and genetically complex tumors (pleomorphic rhabdomyosarcoma and dedifferentiated liposarcoma) to see if there are differences in the pattern of the miRNA clustering and expression.
We asked whether this platform would be sensitive to discriminate differences in expression profiles in a small sample cohort. Second, we asked the question if there were candidate miRNAs that were uniformly differentially expressed across all sarcoma subtypes. Finally, we explored unique expression signatures in different sarcoma subtypes that could be explored further for diagnostic, prognostic, or therapeutic purposes.

\section{MATERIALS AND METHODS Sample Processing and RNA Isolation}

After approval from the Institutional Review Board at The Ohio State University (IRB-approved protocol number 2002H0089), FFPE blocks of 24 sarcomas from 5 histologic types were retrieved including: synovial sarcoma $(n=5)$, myxoid cell liposarcoma $(n=5)$, well-differentiated liposarcoma $(n=5)$, dedifferentiated liposarcoma $(n=5)$, and pleomorphic rhabdomyosarcoma $(n=4)$. All the cases were re-reviewed by an expert soft-tissue pathologist (OHI), and where appropriate, the diagnoses were further validated by additional molecular testing. The cases used for the study are summarized in Table 1. The hematoxylin and eosin-stained sections containing the neoplastic tumoral areas were identified as well as normal non-tumoral areas. Both areas were appropriately marked as a guide for the tissue microarrayer to obtain multiple $1.75 \mathrm{~mm}$ cores simultaneously from the corresponding areas on the FFPE blocks (Figure 1). A total of 24 sarcoma specimens and their matched non-neoplastic samples $(n=24)$ were available for this study. These tissues were all obtained as paired tumor and normal specimens. To minimize preanalytical changes, the tissue cores were all obtained by the same technician in one batch.

In addition, to explore any possible pathogenetic link between normal fat, lipomas, well-differentiated liposarcomas, and dedifferentiated liposarcomas, we expanded the study cohort to include: lipoma $(n=5)$ and normal fat $(n=5)$ as a separate set of controls for lipogenic tumors.

Total RNA was isolated from FFPE human sarcomas and subjacent normal tissue using RecoverAll Total Nucleic Acid Isolation Kit for FFPE tissues (Ambion, CA, USA) according to manufacturer's protocol. Additional glycogen precipitations were performed. RNA concentration and purity were measured using the NanoDrop 2000 (Thermo Fisher Scientific, MA, USA). RNA integrity was analyzed using the Bioanalyzer 2100 (Agilent Technologies, CA, USA).

\section{miRNA Expression Profiling}

The nCounter Human v2 miRNA Expression Assay Kit (NanoString Technologies, WA, USA) ${ }^{20,21}$ was used to profile 800 human miRNAs in human sarcoma and normal tissue cells. Total RNA (100 ng) was used as input material. Preparation of small RNA samples involved the ligation of a specific DNA tag onto the $3^{\prime}$ end of each mature miRNA. These tags were designed to normalize the Tm's of the miRNAs as well as to provide a unique identification 
Table 1 Summary of clinicopathologic features of human sarcomas used in the study

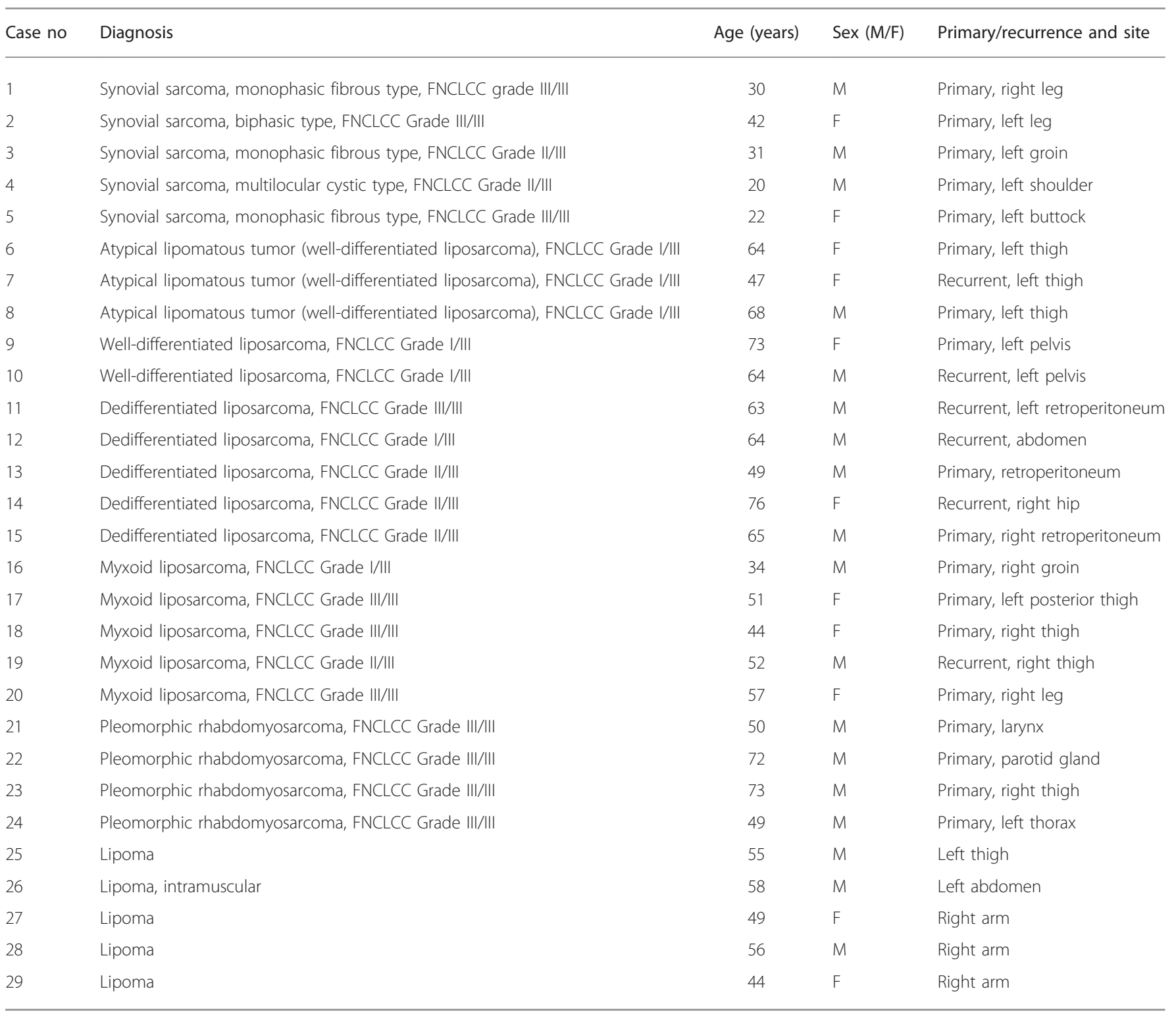

for each miRNA species in the sample. The tagging was accomplished in a multiplexed ligation reaction using reverse-complementary bridge oligonucleotides to direct the ligation of each miRNA to its designated tag. Following the ligation reaction, excess tags and bridges were removed, and the resulting material was hybridized with a panel of miRNA: tag-specific nCounter capture and barcoded reporter probes.

Hybridization reactions were incubated at $64^{\circ} \mathrm{C}$ for a minimum of $18 \mathrm{~h}$. Hybridized probes were purified to remove excess capture and reporter probes and immobilized on a streptavidin-coated cartridge using the nCounter Prep Station (NanoString Technologies). The nCounter Digital Analyzer (NanoString Technologies) was used to count individual fluorescent barcodes and quantify target RNA molecules present in each sample. For each assay, a high-density scan (600 fields of view) was performed.

\section{miRNA Expression Profiling}

The nCounter Human v2 miRNA Expression Assay Kit ${ }^{17,18}$ was used to profile 800 human miRNAs in sarcoma and normal tissue. Total RNA (100 ng) input was used per sample. Expression analysis was conducted at the Ohio State University Nucleic Acid Core Facility according to the manufactory's protocol. MiRNAs were quantified as counts by the nCounter Digital Analyzer.

\section{Statistical Analysis}

Data analysis was conducted at the Ohio State University Comprehensive Cancer Center Biostatistics Core. The miRNAs were filtered out if counts were $<32$ across $90 \%$ of the samples. About 596 miRNAs remained and were normalized by the geometric means. Heat maps were generated by using Multi Experiment Viewer ( $\mathrm{MeV}$ 4.9). 
a

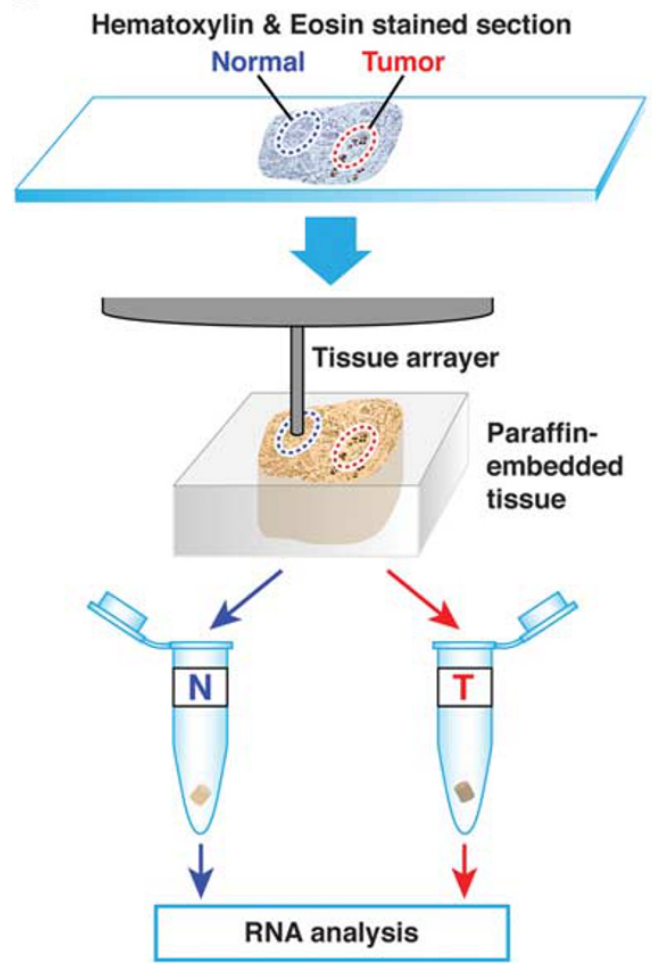

b
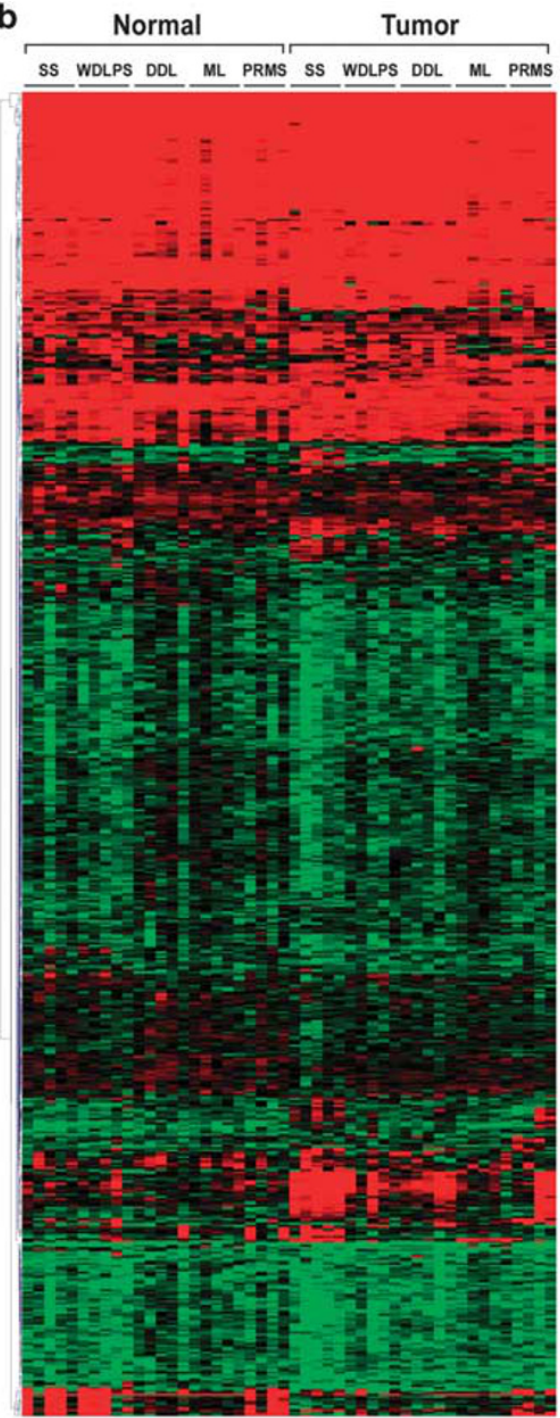

Figure 1 Novel method of comparison shows segregation of peritumoral 'normal' and tumor tissue. (a) A schematic showing a previously validated novel method of obtaining tumoral and non-tumoral tissue from the same patient. 'Normal' tissue (N) was identified subjacent to the tumor (T) on FFPE archival material using hematoxylin and eosin-stained sections as a guide. The tissue microarrayer obtained cores from corresponding areas on formalinfixed paraffin-embedded blocks, and total RNA was subsequently isolated. (b) Heat map with supervised hierarchical clustering with all 'normal' tissues on the left and tumor on the right. Each row represents median-centered expression of a separate miRNA. Red indicates higher expression; green indicates lower expression. Only miRNA showing expression above noise levels ( $>32$ ) across $90 \%$ of the samples were included. DDL, dedifferentiated liposarcoma; FFPE, formalin-fixed paraffin embedded; ML, myxoid liposarcoma; PRMS, pleomorphic rhabdomyosarcoma; SS, synovial sarcoma; WDLPS, well-differentiated liposarcoma.

Hierarchical clustering method was used to cluster miRNAs according to the Euclidean distance to visualize the global miRNA expression (Figure 1). Differential expression of miRNAs between tumor and matched normal samples was tested by using a paired $t$-test for each tumor subtype, while miRNA expression from lipoma and normal fat was tested by a two-sample $t$-test. Smoothing method was applied to improve variance estimates in the tests. ${ }^{22}$ The trend of miRNA expression of normal fat, lipoma, well-differentiated liposarcoma, and dedifferentiated liposarcoma was analyzed by a general linear model. The expected false positive rate was controlled at 0.01 (one false positive out of 100 tests). ${ }^{23}$ The miRNAs showing statistical significant increasing or decreasing trends were chosen for heat map (Figure 4). SAS 9.3 (SAS, Cary, NC) and R 3.0.1 were used for data analysis.

\section{qRT-PCR Analysis}

The NanoString nCounter miRNA data analysis was validated using Life Technologies' single tube TaqMan miRNA assay on the same samples as before. All reagents, primers, and probes were obtained from Life Technologies (CA, USA). Normalization was performed with RNU24. Reverse 
Transcriptase Reactions and Real-Time PCR were performed according to the manufacturer's protocol. All RT reactions, including no-template controls and RT minus controls, were run in a GeneAmp PCR 9700 Thermocycler (Life Technologies). Gene expression levels were quantified using the StepOnePlus Real-Time PCR system (Life Technologies). Comparative real-time PCR was performed in triplicate, including no-template controls. Relative expression was calculated using the comparative $\mathrm{C}_{\mathrm{t}}$ method.

\section{RESULTS \\ miRNA Expression Profiles of Various Sarcomas are Distinctly Different from the Surrounding Peritumoral Normal Controls}

Until now, studies have compared tumor tissue with normal tissue from separate healthy control subjects. Our work introduced a method of comparison wherein the normal tissues came from each subject themselves instead of from random subjects. These control tissues were drawn from normal tissue subjacent to the tumor and were tested to ensure that control cells were indeed tumor-free by an expert soft-tissue pathologist. With this technique, both the control and tumor samples came from the patient biopsy, which offered increased sensitivity and accuracy of analysis by eliminating confounding factors (Figure 1a).

Next, we used a miRNA platform to study the comparative global miRNA expression signatures between tumor and normal areas. Following the screen, we applied hierarchical clustering to the miRNA expression data (Figure 1b). Results showed global differences in expression between these two groups. Of note, the heat map showed that in tumor versus normal tissue, there were more miRNAs that were overexpressed than underexpressed, respectively. In fact, we found the expression levels of 189 miRNAs to be statistically significantly increased in tumors when compared with normal tissue $(P<0.01)$, and the expression levels of 77 miRNAs to be decreased $(P<0.01)$ (Table 2, Supplementary Table 1).

\section{MyomiRs are Underexpressed in Well-Differentiated Liposarcoma and Synovial Sarcoma}

Quite unexpectedly, we found that a class of skeletal muscle-related miRNAs, referred to as myomiRs (miR-1, miR-133, and miR-206), was significantly underexpressed in well-differentiated liposarcomas. These myomiRs have been shown to function as tumor suppressor miRNAs in rhabdomyosarcoma. However, their differential expression has never been described before in other non-myogenic soft-tissue sarcoma subtypes to our knowledge. In well-differentiated liposarcomas, miR-1 and miR-133a appeared to be the most underexpressed compared with miR-206 (Figure 1b, Supplementary Figure 1). MiR-1 and miR-133a were decreased by 125 - and 167 -fold, respectively, when compared with their adjacent normal tissue (Figure 2a). These two miRNAs showed the lowest fold change (fc) differences in the entire study. MiR-133b was also underexpressed in synovial sarcoma $(f \mathrm{c}=0.052)$ (Figure $2 \mathrm{a})$. To further explore the relevance of this finding, we compared our findings with other published works on miRNA expression profile in rhabdomyosarcoma and compiled expression data for miR-1, miR-133a, miR-133b, and miR-206 from ten studies that compared rhabdomyosarcoma with normal skeletal muscle (Supplementary Table 2). ${ }^{9-13,24-28}$ The prior studies showed underexpression of miR-1 and miR-133a (if examined) in all nine studies and variable expression of miR-133b and miR-206 across the comparisons of rhabdomyosarcoma to normal skeletal muscle. The underexpression of the myomiRs miR-1, miR-133a, and miR-206 in well-differentiated liposarcoma was validated using qRT-PCR (Figures $2 \mathrm{~b}$ and $\mathrm{d}$ ).

\section{Candidate miRNAs May be Unique to Different Sarcoma Types}

It became immediately apparent that hierarchical clustering analyses were very tight among synovial sarcoma and myxoid liposarcoma, and least in the pleomorphic rhabdomyosarcoma group. We identified statistically significant increased or decreased expression of miRNA between tumor and subjacent normal tissue (Table 2, Supplementary Table 1). Intriguingly, we found tight clustering of the data even with the small number of samples $(n=5)$ for each subtype. Pleomorphic rhabdomyosarcoma did not show clustering as tight as with the other subtypes.

Synovial sarcoma had the most number of differentially expressed miRNAs, with 99 overexpressed miRNAs and 24 underexpressed miRNAs. Of the 99 overexpressed miRNAs in synovial sarcoma, miR-9-5p and miR-376c had the highest fold changes ( $\mathrm{fc}=30$ and 22). From the 24 underexpressed miRNAs, miR-133b and miR-378 had the lowest fold changes $(f \mathrm{f}=0.052$ and 0.047$)$. Three members of the miR-200 family (miR-200a, miR-200b, and miR-429) were significantly overexpressed ( $\mathrm{fc}=8.73,18.8$, and 3.94 ), and two members of the miR-183 family (miR-182 and miR-183) were also significantly overexpressed $(\mathrm{fc}=5.48$ and 4.04$)$. In addition, miR-26a, miR-199a-3p, miR-199b-3p, miR-199a-5p, miR-127-3p, miR-376a, and miR-34a were overexpressed $\quad\left(\mathrm{fc}_{\mathrm{c}}=2.72, \quad 17.21, \quad 13.14, \quad 13.64, \quad 16.84\right.$, and 5.76 ), while miR-145 was significantly underexpressed $(\mathrm{fc}=0.13)$.

Myxoid liposarcoma had 21 and 7 overexpressed and underexpressed miRNAs respectively. In this subtype, miR-135b and miR-181a had the highest fold changes $\left(f_{c}=17.395\right.$ and 13.167). MiR-29b and miR-150 were the most underexpressed $\left(f_{c}=0.362\right.$ and 0.396 ) in these tumors. Similar to synovial sarcoma, miR-9-5p was significantly overexpressed when comparing myxoid liposarcoma with normal tissue $(\mathrm{fc}=12.07)$, and miR-145 was again significantly underexpressed $(\mathrm{fc}=0.42)$.

Well-differentiated liposarcoma had 11 overexpressed miRNAs and 35 underexpressed miRNAs, making it the only 
Table 2 Distinct miRNA expression signatures in sarcoma subtypes with top 5 miRs shown

\begin{tabular}{lcc}
\hline miR & Fold change & P-value \\
\hline $\begin{array}{l}\text { Synovial sarcoma } \\
\text { Overexpressed miRs }\end{array}$ & & \\
hsa-miR-9-5p & 30.00 & $4.06 \mathrm{E}-06$ \\
hsa-miR-376c & 22.01 & $2.54 \mathrm{E}-06$ \\
hsa-miR-200b-3p & 18.79 & 0.00020257 \\
hsa-miR-199a-3p+hsa-miR-199b-3p & 17.21 & $1.89 \mathrm{E}-07$ \\
hsa-miR-376a-3p & 16.84 & $1.08 \mathrm{E}-06$ \\
& & \\
& & \\
Underexpressed miRs & & \\
hsa-miR-145-5p & 0.13 & $5.59 \mathrm{E}-05$ \\
hsa-miR-150-5p & 0.09 & $4.72 \mathrm{E}-06$ \\
hsa-miR-378 $\mathrm{g}$ & 0.08 & 0.006042798 \\
hsa-miR-133b & 0.05 & 0.0029808 \\
hsa-miR-378a-3p+hsa-miR-378i & 0.05 & 0.00598704
\end{tabular}

Well-differentiated liposarcoma

Overexpressed miRs
hsa-miR-199b-5p
hsa-miR-4286
hsa-miR-382-5p
hsa-miR-335-5p
hsa-miR-409-3p

Underexpressed miRs

hsa-miR-195-5p

hsa-miR-378 g

hsa-miR-27b-3p

hsa-miR-1

hsa-miR-133a

Pleomorphic rhabdomyosarcoma

Overexpressed miRs
hsa-miR-21-5p
hsa-miR-221-3p
hsa-miR-532-5p
hsa-miR-660-5p
hsa-miR-362-3p

Underexpressed miRs

hsa-miR-203

hsa-miR-520 h

hsa-miR-499a-5p

hsa-miR-145-5p

0.000585925
0.002059675

$1.51 \mathrm{E}-05$

0.004704059

0.002629203

0.00386825

0.004733932

0.001887109

0.006071633

0.000415368
Table 2 Continued

\begin{tabular}{lcc}
\hline miR & Fold change & P-value \\
\hline Myxoid liposarcoma & & \\
Overexpressed miRs & & \\
hsa-miR-135b-5p & 17.40 & 0.000328151 \\
hsa-miR-181a-5p & 13.17 & $2.88 \mathrm{E}-05$ \\
hsa-miR-9-5p & 12.07 & $8.54 \mathrm{E}-06$ \\
hsa-miR-193a-3p & 5.53 & $3.41 \mathrm{E}-05$ \\
hsa-miR-106b-5p & 4.67 & 0.001865448 \\
& & \\
Underexpressed miRs & & 0.000130298 \\
hsa-miR-296-5p & 0.44 & 0.001467579 \\
hsa-miR-23b-3p & 0.43 & 0.005388871 \\
hsa-miR-145-5p & 0.42 & 0.001079821 \\
hsa-miR-150-5p & 0.40 & 0.36 \\
hsa-miR-29b-3p & &
\end{tabular}

Dedifferentiated liposarcoma

Overexpressed miRs

0.000975996

3.27E- 05

0.009735526

0.006525381

hsa-miR-26a-5p

22.99

0.000432446

hsa-miR-21-5p

11.82

2.87E- 06

hsa-miR-199a-5p

10.70

$8.19 E-06$

hsa-miR-199a-3p+hsa-miR-199b-3p

9.33

0.000124533

hsa-miR-376c

8.75

5.77E- 05

Underexpressed miRs

hsa-miR-144-3p

0.45

0.000720255

hsa-miR-4516

hsa-miR-139-3p

0.44

$3.52 \mathrm{E}-05$

hsa-miR-302c-3p

0.44

2.47E- 05

$1.34 \mathrm{E}-06$

0.001219054

0.001211507

subtype of sarcoma to have more underexpressed miRNAs than overexpressed miRNAs. The fold changes were more pronounced in the underexpressed miRNAs than in the overexpressed miRNAs. The two miRNAs with the highest fold change differences were miR-199b ( $f c=5.829)$ and miR-4286 ( $\mathrm{fc}=5.807)$. MiR-146b, miR-337, and miR-382 were overexpressed ( $\mathrm{fc}=2.09,2.23$, and 3.34). The two miRNA with the lowest fold change differences were miR-1 ( $\mathrm{fc}=0.008)$ and $\operatorname{miR}-133 \mathrm{a}(\mathrm{fc}=0.006)$. All three mature members of the miR-29 family (miR-29a, -29b, and -29c) were underexpressed $(\mathrm{fc}=0.19,0.21$, and 0.11 ).

On the other hand, dedifferentiated liposarcoma had 46 overexpressed miRNAs and 7 underexpressed miRNAs. Accordingly, the fold changes were more prominent in the 
a

\begin{tabular}{|c|c|c|c|c|c|c|c|c|c|c|}
\hline \multicolumn{5}{|c|}{ Normal } & \multicolumn{5}{|c|}{ Tumor } & \\
\hline SS & WDLPS & DDL & ML & PRMS & SS & WDLPS & DDL & ML & PRMS & \\
\hline
\end{tabular}

b

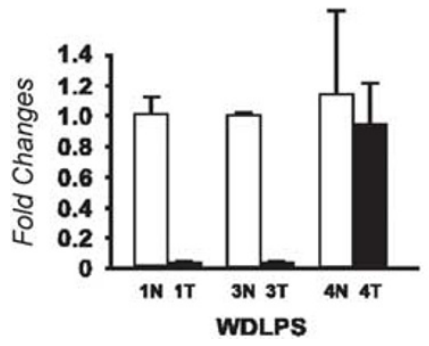

c

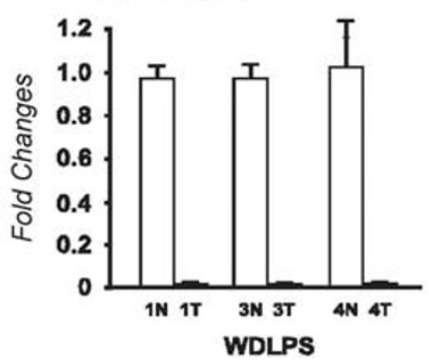

d

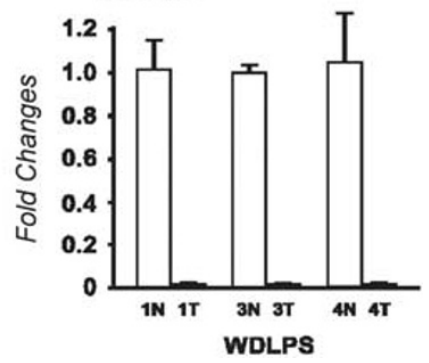

Figure 2 MyomiRs (miR-1, miR-133a, miR-133b, and miR-206) are underexpressed in well-differentiated liposarcoma and synovial sarcoma. (a) Heat map highlighting myomiRs underexpression in well-differentiated liposarcoma and synovial sarcoma shown taken from Figure 1b. MiR-133a, miR-1, miR-133b, and miR-206 were found to be clustered close to each other. MiR-133a and miR-1 were next to each other, and miR-133b and miR-206 were next to each other. All four miRs are shown together. (b-d) Quantitative Real-Time PCR validation analysis of miR-206 (b), miR-133a (c), and miR-1 (d) in well-differentiated liposarcoma. Quantitative real-time PCR was performed in triplicate, including no-template controls. Relative expression was calculated using the comparative $C_{t}$ method. DDL, dedifferentiated liposarcoma; miR, miRNA; ML, myxoid liposarcoma; PRMS, pleomorphic rhabdomyosarcoma; SS, synovial sarcoma; WDLPS, well-differentiated liposarcoma.

overexpressed miRNAs than in the underexpressed miRNAs. MiR-26a, miR-21, and miR-199a had the highest fold changes ( $\mathrm{fc}=22.994,11.821$, and 10.695), while miR-187 and miR-302c had the lowest fold changes $\left(\mathrm{fc}_{\mathrm{c}}=0.382\right.$ and 0.408). Five miRNAs (miR-146b, miR-199b, miR-337, miR-382, and miR-409) out of 11 miRNAs that were overexpressed in well-differentiated liposarcoma were similarly overexpressed in dedifferentiated liposarcoma $(\mathrm{fc}=3.15,4.22,6.17,3.55,3.14)$. In addition, miR-21, miR-26a, miR-34a, miR-127-3p, miR-199b-5p, miR-376a, miR-376c, and miR-377 were overexpressed $(\mathrm{fc}=11.8,23$, $5.72,4.83,4.22,6.51$, and 8.75), while miR-144-3p was significantly underexpressed $(\mathrm{fc}=0.45)$. Thus, more miRNAs were overexpressed in dedifferentiated than in well-differentiated liposarcoma. Similarly to well-differentiated liposarcoma, miR-146b, miR-199b-5p, miR-337, and miR-382 were overexpressed in the dedifferentiated subtype $(\mathrm{fc}=3.15$, $4.22,6.17,3.55)$. In addition, miR-21, miR-26a, miR-34a, miR-127-3p, miR-199b-5p, miR-376a, miR-376c, miR-377, and miR-409 were overexpressed $\left(\mathrm{f}_{\mathrm{c}}=11.8,23,5.72,4.83\right.$, $4.22,6.51,8.75$, and 3.14 ), while miR-144-3p was significantly underexpressed $(\mathrm{fc}=0.45)$. Noteworthy, from the 46 overexpressed miRNAs in dedifferentiated liposarcoma, only 1 (let-7i) is in the 12q13 15 amplification that characterizes well-differentiated and dedifferentiated liposarcomas.

Pleomorphic rhabdomyosarcoma had 12 overexpressed miRNAs and 4 underexpressed miRNAs. In pleomorphic rhabdomyosarcoma tissue, miR-21 had the highest fold change difference $(\mathrm{fc}=11.145)$, and $\mathrm{miR}-221$ had the next highest with a fold change of 6.813 . The two lowest fold change differences were miR-145 $(\mathrm{fc}=0.287)$ and $\mathrm{miR}-499 \mathrm{a}$ $(\mathrm{fc}=0.354)$.

\section{MiR-145 is Underexpressed in Synovial Sarcoma, Pleomorphic Rhabdomyosarcoma, and Well-Differentiated Liposarcoma}

We did not find any miRNA that was consistently overexpressed or underexpressed in the same direction in all sarcoma types. MiR-145 was the only miRNA to be similarly underexpressed in three out of six sarcoma subtypes. The underexpression of miR-145 was validated by qRT-PCR in synovial sarcoma, pleomorphic rhabdomyosarcoma, and well-differentiated liposarcoma tumors (Figure 3).

\section{Differential miRNA Expression Between Normal Fat, Lipomas, Well-Differentiated, and Dedifferentiated Liposarcoma}

A striking finding made with our screen was that there was a progressive differential expression of miRNAs from a sample cohort of normal fat to lipomas to well-differentiated and finally to dedifferentiated liposarcoma (Figure 4a). We performed a linear trend test between normal fat, lipomas, well-differentiated, and dedifferentiated liposarcoma (Figure 4). There were vastly more overexpressed miRNAs than underexpressed miRNAs, with 87 overexpressed miRNA and 6 underexpressed miRNAs $(P$-values for trend tests $<0.01)$. The six underexpressed miRNAs in the linear trend test were miR-144, miR-149, miR-497, miR-579, miR-612, and miR-652. The underexpression of miR-652 was validated by qRT-PCR using the average of the normal fat $\mathrm{Ct}$ values as reference (Figure 4d). 


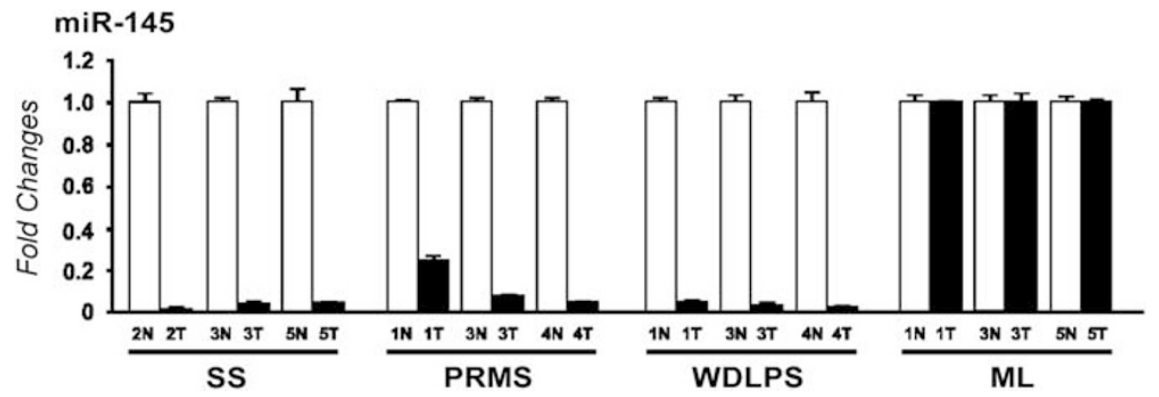

Figure 3 MiR-145 is underexpressed in three sarcoma subtypes. Quantitative Real-Time PCR analysis of miR-145 in synovial sarcoma, pleomorphic rhabdomyosarcoma, and well-differentiated liposarcoma. Tests were performed as in Figures $2 \mathrm{~b}$ and d. ML, myxoid liposarcoma; PRMS, pleomorphic rhabdomyosarcoma; SS, synovial sarcoma; WDLPS, well-differentiated liposarcoma.

\section{DISCUSSION}

The inherent heterogenous landscape of sarcomas continues to present a therapeutic challenge because of its complex molecular and phenotypic diversity. Emerging evidence appears to implicate miRNAs as contributors to the pathogenesis of several hematological and solid organ malignancies. The objective of this study was to explore miRNA expression in a variety of human sarcoma subtypes. We used a selective miRNA screening platform to perform an extensive miRNA microarray expression profiling of 24 sarcomas comprising 5 different histologic subtypes and subjacent non-tumoral tissues. We first asked whether this platform would be sensitive to discriminate differences in expression profiles in a small sample cohort. Our data highlight the exquisite global differences between paired tumoral and normal areas from a small sample size with hierarchical clustering analysis, validating the sensitivity of this platform. The exquisitely high level of sensitivity of the Nanostring platform for the selected cohort of soft-tissue sarcomas with such small sample size is quite remarkable.

The distinct differential expression of candidate miRNAs appeared to cluster tightly mostly in the sarcomas with simple reciprocal translocations like myxoid liposarcoma and synovial sarcoma subtypes and much less so in the genetically complex ones such as pleomorphic rhabdomyosarcoma. The variable expression of miRNA in pleomorphic rhabdomyosarcoma might be due to the non-recurrent complex derangement of the genome. Conceivably, these may be reflective of the implicit differences in the biology of these tumors with potential implications for diagnosis, therapeutics, and prognosis.

We did not find any candidate miRNA that was uniformly differentially expressed across all subtypes, consistent with the inherent heterogeneity of these tumors. We found miR-145 to be the most uniformly differentially expressed miRNA across the subtypes of sarcoma. In our study, miR-145 was underexpressed in synovial sarcoma, pleomorphic rhabdomyosarcoma, and well-differentiated liposarcoma. MiR-145 is a microRNA of growing interest, which has been implicated to have a critical role in the tumorigenesis of many different solid organ malignancies. In oral squamous cell carcinoma, miR-145 is downregulated. ${ }^{29}$ Functionally, miR-145 inhibits cell growth by targeting c-myc and cdk6 (ref. 30), and affects the risk of esophageal squamous cell carcinoma through co-regulating fascin homolog $1 .^{31}$ MiR-145 is suppressed in renal cell carcinoma and targets metalloprotease ADAM17. ${ }^{32}$ This miRNA is downregulated in glial tumors and regulates glioma cell migration by targeting connective tissue growth factor. ${ }^{33}$ In breast cancer cells, miR-145 mediates the epithelial to mesenchymal transition by targeting Oct4. ${ }^{34}$ MiR-145 is regulated by DNA methylation and p53 gene mutation in prostate cancer. ${ }^{35}$ Another tumor suppressor function of miR-145 is that it inhibits tumor angiogenesis and growth by targeting N-RAS and VEGF. ${ }^{36}$ Thus, our results suggest that miR-145 might function similarly in sarcomas as epithelial tumors. Additional mechanistic studies are warranted to explore a broader role of this miRNA in these sarcomas.

We next explored unique expression signatures in different sarcoma subtypes that could be examined further for diagnostic, prognostic, or therapeutic purposes. Unexpectedly, we found for the first time that in well-differentiated liposarcoma and synovial sarcoma, miR-1, miR-133a, and miR-206 (known as myomiRs) were significantly underexpressed, results that we validated by qRT-PCR. These miRNAs are muscle-specific and have a significant role in skeletal muscle development and proliferation. ${ }^{37}$ They are regulated by myogenic transcription factors such as MyoD, Myf5, myogenin, MRF4, and Mef2, and are necessary for skeletal muscle formation. ${ }^{9}$ Through various mechanisms, myomiRs activate muscle genes and coordinate myoblasts to leave the cell cycle to fuse into multinucleated myotubes. ${ }^{38}$ These myomiRs have also been implicated in cancer and muscle diseases. ${ }^{39}$ MiR-1, miR-133a, and miR-133b have been reported as significantly under-represented in leiomyosarcoma ${ }^{40}$ and rhabdomyosarcoma. ${ }^{11}$ There are reports of miR-1 in epithelial malignancies including non-small cell lung cancer, head and neck squamous cell carcinoma, and hepatocellular carcinoma, ${ }^{41-43}$ but this has not yet been 
a

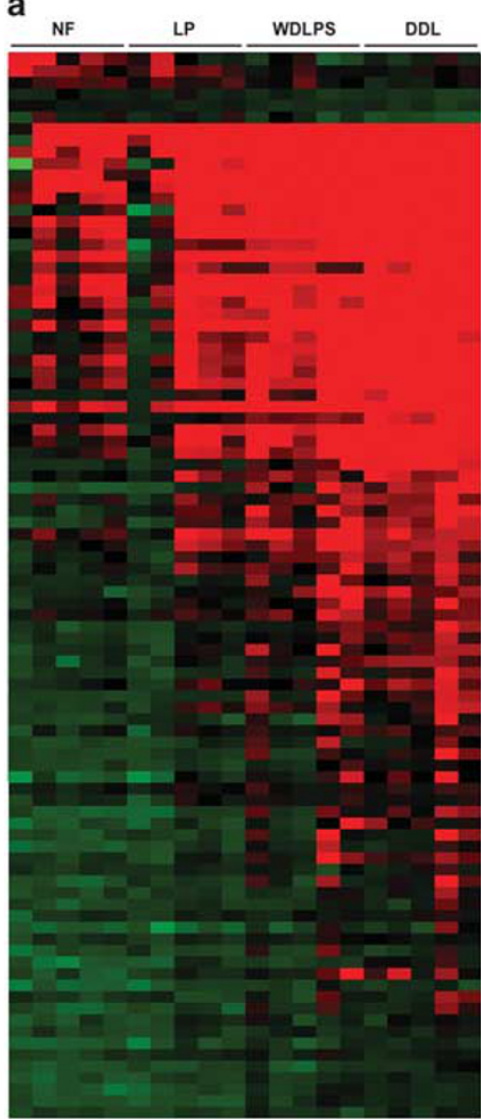

b
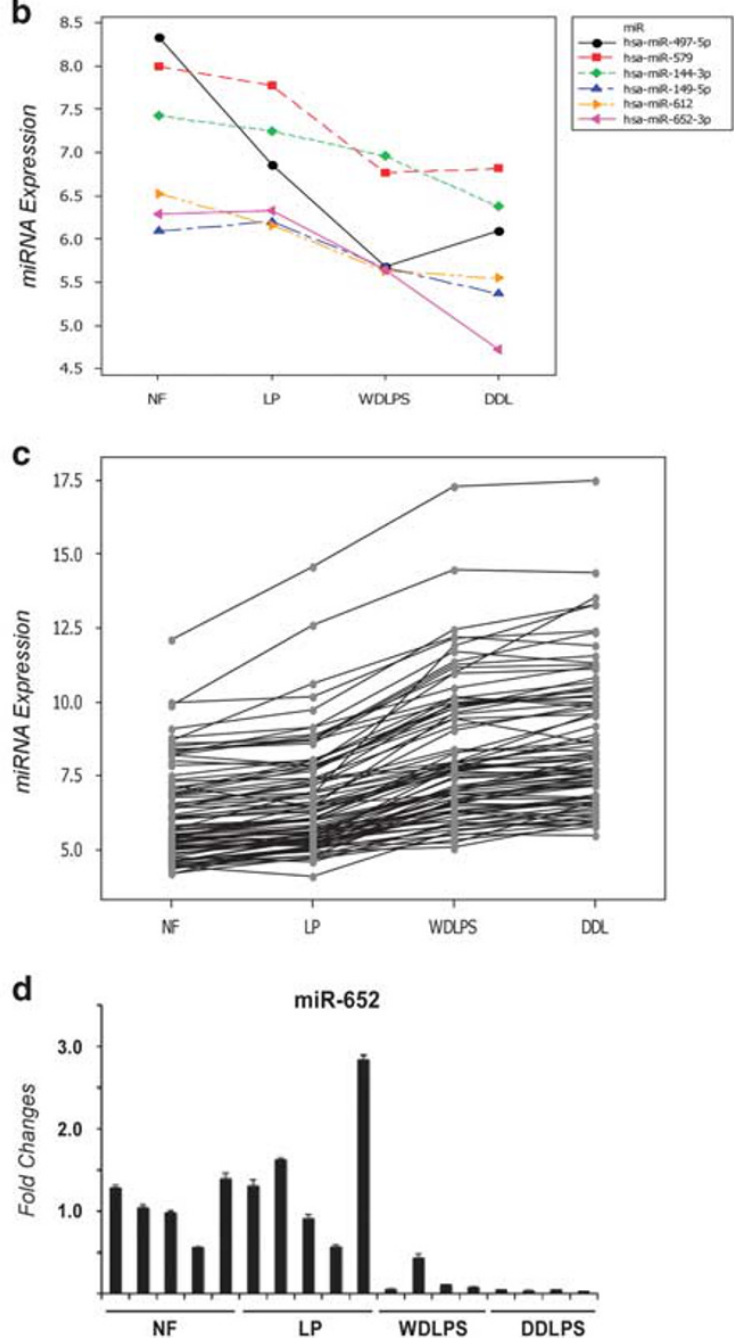

Figure 4 Differential miRNA expression between normal fat, lipomas, well-differentiated liposarcoma, and dedifferentiated liposarcoma. (a) Heat map illustration showing progressive increase or decrease in miR expression from normal fat and lipoma to well-differentiated liposarcoma and dedifferentiated liposarcoma. Red indicates higher expression level; green indicates lower expression level. (b and c) Linear trend with the tissue type on the horizontal axis and mean expression of miRNA within each type on the vertical axis shows that expression levels of candidate miRNAs progressively decrease (b) or increase (c) from normal fat, lipomas, well-differentiated liposarcoma to dedifferentiated liposarcoma. (b). Six candidate miRs: has-miR-497-5p, has-miR-579, has-miR-144-3p, hsa-miR-149-5p, has-miR-612, and has-miR-652-3p showing downward trend from normal fat, lipoma, well-differentiated liposarcoma to dedifferentiated liposarcoma. (d) Quantitative Real-Time PCR analysis of miR-652 across normal fat, lipomas, well-differentiated liposarcoma, and dedifferentiated liposarcoma. Fold changes were determined compared with the average normal fat $C_{t}$ value. $D D L$, dedifferentiated liposarcoma; LP, lipoma; NF, normal fat; WDLPS, well-differentiated liposarcoma.

reported in non-myogenic sarcomas. The downregulation of miR-1 appeared to correlate inversely with the upregulation of Met in both non-small cell lung cancers and hepatocellular carcinomas. In head and neck squamous cell carcinomas, miR-1 acts as a tumor suppressor by directly targeting transgelin-2, suggesting possible oncogenic pathways in the pathogenesis of head and neck squamous cell carcinoma. ${ }^{42}$ Another miRNA of interest is miR-29. This miR functions as a positive regulator of myogenesis through feedback inhibition of YY1 and also functions as a tumor suppressor in rhabdomyosarcoma. ${ }^{9}$ In our study, miR-29a, miR-29b, and miR-29c were underexpressed in well-differentiated liposarcoma. MiR-29a was overexpressed in dedifferentiated liposarcoma, and miR-29b was underexpressed in myxoid liposarcoma. Our results imply that these miRNAs might be functioning as tumor suppressors in both muscle and non-muscle derived tumors. The underexpression pattern of myomiRs in well-differentiated liposarcoma was similar to prior studies of rhabdomyosarcoma because miR-1 and miR-133a showed the strongest underexpression.

We further explored unique expression signatures by examining differential miRNA expression in well-differentiated liposarcoma and dedifferentiated liposarcoma. We first contrasted our results with the work of Ugras et al ${ }^{44}$ which 
compared these two sarcoma subtypes with normal fat. They identified seven miRNAs to be overexpressed in well-differentiated liposarcoma compared with normal fat tissue samples, as we found 11 overexpressed miRNAs in this same tumor type. Only one miRNA was overexpressed in both data sets (miR-199b-3p), possibly secondary to the small sample size in our study. Interestingly, all seven miRNAs that were previously identified to be overexpressed in well-differentiated liposarcoma compared with normal fat were overexpressed in our synovial sarcoma analysis. In dedifferentiated liposarcoma, they identified 15 miRNAs to be overexpressed. This was consistent with 12 of the 46 overexpressed miRNAs in our data. In both subtypes, we found more overexpressed microRNAs than prior studies, perhaps in part due to our experimental design of taking tumor and normal from the same patient.

Past studies have shown that the relationship between well-differentiated liposarcoma and dedifferentiated liposarcoma is unclear. In our study, we observed a linear trend using a small cohort of normal fat, lipoma, well-differentiated liposarcoma, and dedifferentiated liposarcoma samples. Six miRNAs (miR-497, miR-579, miR-144-3p, miR-149-5p, miR-612, and miR-652-3p) showed a downward trend. MiR-144 has recently been shown to be downregulated in bladder cancer, which leads to loss of inhibition of zeste homolog 2 and subsequent activation of Wnt/ $\beta$-catenin signaling with ensuing cellular proliferation. ${ }^{45}$ Xie et $a l^{46}$ showed that miR-497 is underexpressed in hepatocellular carcinoma and that this miRNA is a negative regulator of checkpoint kinase 1 , which functions in cell cycle arrest after DNA damage. The roles of these tumor suppressor miRNAs have not yet been explored in the biology of adipocytic neoplasms. From these trends, the pathogenesis of well-differentiated liposarcoma may be more related to loss of tumor suppressors, while the pathogenesis of dedifferentiated liposarcoma may be relevant to oncomiR gain of function. Dedifferentiated liposarcoma showed overexpression of 46 miRNAs, which was an increase compared with the 11 miRNAs overexpressed in well-differentiated liposarcoma. Let-7i was overexpressed in dedifferentiated liposarcoma, and was the only miRNA overexpressed, that is, in the 12 q13 $~ 15$ amplification that characterizes well-differentiated liposarcoma and dedifferentiated liposarcoma. Five miRNAs (miR-146b, -199b, -337, -382, and -409) were found to be overexpressed in both well-differentiated liposarcoma and dedifferentiated liposarcoma compared with subjacent normal tissue, but not in lipoma or in normal fat. Three of these miRNAs (miR-146b, miR-199b, and miR-382) were also overexpressed in the progression analysis. Taken together, our data suggest that differential expression of these miRNAs may be involved in the evolution and malignant transformation of liposarcoma.

In summary, our results identified numerous differentially expressed miRNAs that warrant further study. Our highly selective platform uncovered a class of putative tumor suppressor myomiRs in two subtypes of non-myogenic soft-tissue sarcomas.
We also identified that miRNAs form linear trends of miRNA expression from normal fat to well-differentiated liposarcoma and dedifferentiated liposarcoma that may have biologic, diagnostic, therapeutic, or prognostic implications.

Supplementary Information accompanies the paper on the Laboratory Investigation website (http://www.laboratoryinvestigation.org)

\section{ACKNOWLEDGMENTS}

We thank Raphael E Pollock and Kate Lynn J Bill, for their critical review of this manuscript. This work was supported in part by NIH grants, R01 CA143082 and P01 CA163995 to DCG and an OSU College of Medicine Bennett research scholarship to PYY.

\section{DISCLOSURE/CONFLICT OF INTEREST}

The authors declare no conflict of interest.

1. Clark MA, Fisher C, Judson I et al. Soft-tissue sarcomas in adults. N Engl J Med 2005;353:701-711.

2. Miska EA. How microRNAs control cell division, differentiation and death. Curr Opin Genet Dev 2005;15:300-306.

3. Shivdasani RA. microRNAs: regulators of gene expression and cell differentiation. Blood 2006;108:3646-3653.

4. Calin GA, Svignani C, Dumitru CD et al. Human microRNA genes are frequently located at fragile sites and genomic regions involved in cancers. Proc Natl Acad Sci USA 2004;101:2999-3004.

5. Kent OA, Mendell JT. A small piece in the cancer puzzle: microRNAs as tumor suppressors and oncogenes. Oncogene 2006;25: 6188-6196.

6. Nana-Sinkam SP, Fabbri M, Croce CM. microRNAs in cancer: personalizing diagnosis and therapy. Ann NY Acad Sci 2010;1210: 25-33.

7. Drury R, Verghese ET, Hughes TA. The roles of microRNAs in sarcomas. J Pathol 2012;227:385-391.

8. Subramanian S, Kartha RV. microRNA-mediated gene regulations in human sarcomas. Cell Mol Life Sci 2012;69:693571-693585.

9. Wang H, Garzon R, Sun H et al. NF-kappB-YY1-miR-29 regulatory circuitry in skeletal myogenesis and rhabdomyosarcoma. Cancer Cell 2008;14:369-381.

10. Subramanian $\mathrm{S}$, Lui WO, Lee $\mathrm{CH}$ et al. microRNA expression signature of human sarcomas. Oncogene 2008;27:2015-2026.

11. Taulli R, Bersani F, Foglizzo $V$ et al. The muscle-specific microRNA miR-206 blocks human rhabdomyosarcoma growth in xenotransplanted mice by promoting myogenic differentiation. J Clin Invest 2009;119:2366-2378.

12. Yan D, Dong Xda E, Chen X et al. microRNA-1/206 targets c-Met and inhibits rhabdomyosarcoma development. J Biol Chem 2009;284: 29596-29604.

13. Missiaglia E, Shepherd CJ, Patel S et al. microRNA-206 expression levels correlate with clinical behaviour of rhabdomyosarcomas. $\mathrm{Br} J$ Cancer 2010;102:1769-1777.

14. Miyachi M, Tsuchiya $K$, Yoshida $\mathrm{H}$ et al. Circulating muscle-specific microRNA, miR-206, as a potential diagnostic marker for rhabdomyosarcoma. Biochem Biophys Res Commun 2010;400:89-93.

15. Carbonell J, Alloza E, Arce $P$ et al. A map of human microRNA variation uncovers unexpectedly high levels of variability. Genome Med 2012;4: 62.

16. Lukiw WJ. Variability in micro RNA (miRNA) abundance, speciation and complexity amongst different human populations and potential relevance to Alzheimer's disease (AD). Front Cell Neurosci 2013;7: 133.

17. Rawlings-Goss RA, Campbell MC, Tishkoff SA. Global populationspecific variation in miRNA associated with cancer risk and clinical biomarkers. BMC Med Genomics 2014;7:53.

18. Pritchard $\mathrm{CC}$, Cheng $\mathrm{HH}$, Tewari M. microRNA profiling: approaches and considerations. Nat Rev Genet 2012;13:358-369.

19. Balkhi MY, Iwenofu OH, Bakkar $\mathrm{N}$ et al. miR-29 acts as a decoy in sarcomas to protect the tumor suppressor A20 mRNA from degradation by HuR. Sci Signal 2013;6:ra63. 
20. Geiss GK, Bumgarner RE, Birditt $\mathrm{B}$ et al. Direct multiplexed measurement of gene expression with color-coded probe pairs. Nat Biotechnol 2008;26:317-325.

21. Wyman SK, Knouf EC, Parkin RK. Post-transcriptional generation of miRNA variants by multiple nucleotidyl transferases con tributes to miRNA transcriptome complexity. Genome Res 2011;21: 1450-1461.

22. Smyth GK. Linear models and empirial bayes methods for assessing differential expression in microarray experiments. Stat Appl Genet Mol Biol 2004;3:Article3.

23. Gordon D, Haynes C, Yang $Y$ et al. Linear trend tests for casecontrol genetic association that incorporate random phenotype and genotype misclassification error. Genet Epidemiol 2007;31: 853-870.

24. Ciarapica R, Russo G, Verginelli $F$ et al. Deregulated expression of miR-26a and Ezh2 in rhabdomyosarcoma. Cell Cycle 2009;8: $172-175$.

25. Rao PK, Missiaglia E, Shields L et al. Distinct roles of miR-1 and miR-133a in the proliferation and differentiation of rhabdomyosarcoma cells. FASEB J 2010;24:3427-3437.

26. Li L, Sarver AL, Alamgir $S$ et al. Downregulation of microRNAs miR-1, -206 and -29 stabilizes PAX3 and CCND2 expression in rhabdomyosarcoma. Lab Invest 2012;92:571-583.

27. Sarver AL, Phalak R, Thayanithy $V$ et al. S-MED: sarcoma microRNA expression database. Lab Invest 2010;90:753-761.

28. Megiorni F, Cialfi S, McDowell HP et al. Deep sequencing the microRNA profile of rhabdomyosarcoma reveals down-regulation of miR-378 family members. BMC Cancer 2014;14:880.

29. $\mathrm{Wu} B \mathrm{~L}, \mathrm{Xu} \mathrm{LY}$, Liao LD et al. MiRNA profile of esophageal squamous cell carcinoma: downregulation of miR-143 and miR-145. World J Gastroenterol 2011;17:79-88.

30. Shao Y, Qu Y, Dang S et al. MiR-145 inhibits oral squamous cell carcinoma (OSCC) cell growth by targeting c-Myc and Cdk6. Cancer Cell Int 2013;13:51.

31. Liu R, Liao J, Yang M et al. The cluster of miR-143 and miR-145 affects the risk for esophageal squamous cell carcinoma through coregulating fascin homolog 1. PLoS One 2012;7:e33987.

32. Doberstein K, Steinmeyer N, Hartmetz AK et al. microRNA-145 targets the metalloprotease ADAM17 and is suppressed in renal cell carcinoma patients. Neoplasia 2013;15:218-230.
33. Lee HK, Bier A, Cazacu S et al. microRNA-145 is downregulated in glial tumors and regulates glioma cell migration by targeting connective tissue growth factor. PLoS One 2013;8:e54652.

34. $\mathrm{Hu}$ J, Guo H, Li H et al. MiR-145 regulates epithelial to mesenchymal transition of breast cancer cells by targeting Oct4. PLoS One 2012;7: e45965.

35. Suh SO, Chen Y, Zaman MS et al. microRNA-145 is regulated by DNA methylation and p53 gene mutation in prostate cancer. Carcinogenesis 2011;32:772-778.

36. Zou $C, \mathrm{Xu} Q$, Mao $\mathrm{F}$ et al. MiR-145 inhibits tumor angiogenesis and growth by N-RAS and VEGF. Cell Cycle 2012;11:2137-2145.

37. Chen JF, Mandel EM, Thomson JM et al. The role of microRNA-1 and microRNA-133 in skeletal muscle proliferation and differentiation. Nat Genet 2006:38:228-233.

38. Sabourin LA, Rudnicki MA. The molecular regulation of myogenesis. Clin Genet 2000;57:16-25.

39. $\mathrm{Yu} H, \mathrm{Lu} \mathrm{Y}, \mathrm{Li} \mathrm{Z}$ et al. microRNA-133: expression, function and therapeutic potential in muscle diseases and cancer. Curr Drug Targets 2014;15:817-828.

40. Renner M, Czwan E, Hartmann W et al. microRNA profiling of primary high-grade soft tissue sarcomas. Genes Chromosomes Cancer 2012;51: 982-996.

41. Nasser MW, Datta J, Nuovo G et al. Down-regulation of micro-RNA-1 (miR-1) in lung cancer. Suppression of tumorigenic property of lung cancer cells and their sensitization to doxorubicin-induced apoptosis by miR-1. J Biol Chem 2008;283:33394-33405.

42. Nohata N, Sone $\mathrm{Y}$, Hanazawa $\mathrm{T}$ et al. miR-1 as a tumor suppressive microRNA targeting TAGLN2 in head and neck squamous cell carcinoma. Oncotarget 2011;2:29-42.

43. Datta J, Kutay $\mathrm{H}$, Nasser MW et al. Methylation mediated silencing of microRNA-1 gene and its role in hepatocellular carcinogenesis. Cancer Res 2008;68:5049-5058.

44. Ugras S, Brill E, Jacobsen A et al. Small RNA sequencing and functional characterization reveals microRNA-143 tumor suppressor activity in liposarcoma. Cancer Res 2011;71:5659-5669.

45. Guo Y, Ying L, Tian $Y$ et al. miR-144 downregulation increases bladder cell proliferation by targeting EZH2 and regulating Wnt signaling. FEBS J 2013;280:4531-4538.

46. Xie $Y$, Wei RR, Huang GL et al. Checkpoint kinase 1 is negatively regulated by miR-497 in hepatocellular carcinoma. Med Oncol 2014;31:844. 